\title{
Cosmic-Ray Anisotropy with Seven Years of Data from IceCube and IceTop
}

\section{The IceCube Collaboration ${ }^{\dagger}$}

${ }^{\dagger}$ http://icecube.wisc.edu/collaboration/authors/icrc17_icecube

E-mail: james.bourbeaulicecube.wisc.edu

\begin{abstract}
With data collected over the last seven years, the IceCube Neutrino Observatory at the South Pole has measured both the large- and small-scale anisotropy in the cosmic-ray arrival direction distribution with a high level of significance. In addition to the sidereal anisotropy, we have also measured the solar dipole caused by the orbital motion of the Earth around the Sun. We present the cosmic-ray anisotropy measurement with a full seven years of data and a systematic study of both the sidereal anisotropy and solar dipole.
\end{abstract}

Corresponding authors: James Bourbeau* ${ }^{* 1}$, P. Desiati ${ }^{1}$, J.C. Díaz-Vélez ${ }^{1}$, and S. Westerhoff ${ }^{1}$

${ }^{1}$ Wisconsin IceCube Particle Astrophysics Center (WIPAC) and Department of Physics, University of Wisconsin-Madison, Madison, WI 53706, USA

35th International Cosmic Ray Conference - ICRC2017

10-20 July, 2017

Bexco, Busan, Korea

${ }^{*}$ Speaker. 


\section{Introduction}

Over the last few decades, a number of surface and underground experiments have observed a statistically significant anisotropy in the arrival direction distribution of cosmic rays in the energy range from tens of $\mathrm{GeV}$ to tens of $\mathrm{PeV}$. In the $\mathrm{TeV}$ to $\mathrm{PeV}$ range, measurements of anisotropy have been published by a number of experiments using different detection and analysis techniques, including the Tibet AS $\gamma$ [1], Super-Kamiokande [2, 3], Milagro [4, 5], EAS-TOP [6], MINOS [7], ARGO-YBJ [8], and HAWC [9] experiments in the Northern Hemisphere and IceCube [10, 11, 12, 13] and its surface air shower array IceTop [14] in the Southern Hemisphere.

The angular power spectrum of the arrival direction distribution shows that while most of the power is in the low multipole terms $\left(\ell \leq 4\right.$, corresponding to angular scales greater than $45^{\circ}$ ), features of smaller angular scale down to a few degrees are also present. The relative intensity of the large-scale anisotropy $(\ell \leq 4)$ is at the level of $10^{-3}$, an order of magnitude larger than the intensity of the small-scale structure. Observations below $100 \mathrm{TeV}$ show a structurally consistent large-scale anisotropy with wide relative excess and deficit regions. This structure strongly depends on energy. The amplitude of the anisotropy decreases from $50 \mathrm{TeV}$ to $100 \mathrm{TeV}$. Above $100 \mathrm{TeV}$ the phase of the anisotropy changes and the sky maps now show a wide relative deficit in right ascension with an amplitude that increases with energy until at least $5 \mathrm{PeV}$, where statistics become poor. Recent measurements with the Pierre Auger Observatory at EeV energies show a significant dipole at energies above $8 \mathrm{EeV}$, but no deviation from isotropy at any other scale or at lower energies [15].

While the source of the anisotropy remains unknown, it has been shown that standard diffusive propagation of cosmic rays in the Galaxy from stochastically distributed sources can qualitatively explain the large-scale structure. The phase shift at higher energies potentially indicates a change in the location of the dominant source(s). The small-scale structure can be produced by the interaction of cosmic rays with the turbulent interstellar magnetic field. For a further discussion of possible explanations for the large- and small-scale structure as well as for the energy-dependence of the anisotropy, we refer to the summary and discussion section in [13] and a recent review article [16].

In the Southern Hemisphere, the IceCube Neutrino Observatory has accumulated one of the largest cosmic-ray data sets to date, allowing for a detailed study of the morphology and the timeand energy-dependence of the anisotropy from $\mathrm{TeV}$ to $\mathrm{PeV}$ energies. Located at the geographic South Pole, IceCube comprises a neutrino detector buried in the deep ice (hereafter referred to as the in-ice component) and a surface air shower array, IceTop. IceCube [17] consists of 86 vertical strings containing a total of 5,160 optical sensors, called Digital Optical Modules (DOMs), which are frozen in the ice at depths from 1450 to $2450 \mathrm{~m}$ below the surface. The total instrumented volume is about a cubic kilometer. IceTop [18] consists of 81 surface stations spread over an area of $1 \mathrm{~km}^{2}$. Each station consists of two light-tight tanks with a $1.82 \mathrm{~m}$ inner diameter that are filled with ice to a height of $0.90 \mathrm{~m}$. Each tank hosts two DOMs to detect the Cherenkov light generated by the relativistic particles of the air shower reaching the detector level and traversing the tanks.

With its two components, IceCube detects cosmic rays over a wide range of energies. The in-ice component detects downward-going muons created in air showers initiated by cosmic-ray primaries. According to simulations, the energy of the primary cosmic rays ranges from approximately $10 \mathrm{TeV}$ to $5 \mathrm{PeV}$, where current statistics becomes poor. The trigger rate is modulated by seasonal variations and ranges between 2 and $2.4 \mathrm{kHz}$. The IceTop air shower array detects cosmic 
rays above $400 \mathrm{TeV}$. The median energy is $1.6 \mathrm{PeV}$. This enables IceTop, which mainly measures the electromagnetic component of the air showers, to provide an independent measurement at the high-energy end of the range of the in-ice detector. The trigger rate is $30 \mathrm{~Hz}$.

A detailed study of the large- and small-scale anisotropy with IceCube and IceTop based on six years of data was recently published [13]. Here, we report updated results including an additional year of data, bringing the total data set size to 368 billion cosmic-ray events observed by the in-ice component and 196 million events observed with IceTop. The data were taken between May 2009 and May 2016. In the first two years, the detector was operated in partial detector configurations with 59 and 79 active strings, respectively (IC59/IC79). Subsequent years with the full 86 string detector are labeled IC86-I to IC86-V. The seven year period covered by this analysis allows for a study of a possible time dependence during the first part of the current (24th) solar cycle.

\section{Analysis and Results}

Details about the analysis methods used in this work have been published previously [11, 14, 13]. All sky maps shown here were made using the HEALPix [19] mapping program to pixelize the sky into bins of equal solid-angle. Here, we use a pixel size of $\left(0.84^{\circ}\right)^{2}\left(N_{\text {side }}=64\right)$. The maps are top-hat smoothed with a $5^{\circ}$ angular radius (each pixel's value is replaced with the sum of all pixels within a 5 radius.).

Following [13], we split the in-ice data in nine energy bins according to the number of DOMs hit in the event and the cosine of the reconstructed zenith angle. This results in a sequence of maps with increasing median energy, ranging from $13 \mathrm{TeV}$ for the lowest energy bin to $5.4 \mathrm{PeV}$ for the highest energy bin. For the IceTop data, we only use one energy bin with a median energy of $1.6 \mathrm{PeV}$. Figure 1 shows the sky maps in relative intensity for all nine energy bins in equatorial coordinates. The median energy of the data shown in each map is indicated in the upper left. The maps clearly show the strong dependence of the anisotropy on energy and the change in the morphology above about $100 \mathrm{TeV}$, where the sky maps now show a wide relative deficit from $30^{\circ}$ $120^{\circ}$ in right ascension. The amplitude increases with energy.

To illustrate the energy dependence of the phase and the amplitude of the anisotropy, we fit the set of harmonic functions with $n \leq 3$ to the projection of the two-dimensional relative intensity map in right ascension $\alpha$,

$$
\sum_{n=0}^{3} A_{n} \cos \left[n\left(\alpha-\phi_{n}\right)\right]
$$

where $A_{n}$ is the amplitude and $\Phi_{n}$ is the phase of the $n^{\text {th }}$ harmonic term. Figure 2 shows the amplitude (left) and phase (right) of the dipole moment as a function of energy. The red data point is based on the IceTop data. While the phase agrees well with that of the IceCube data at similar energies, the amplitude of the anisotropy is larger for the IceTop data than for any IceCube energy bin. A possible explanation for the difference could be the different chemical composition of the IceCube and IceTop data sets (see Table 4 in [13]). If the anisotropy is predominantly caused by protons, the lighter composition of the IceTop data could lead to a stronger dipole amplitude.

The data used in this analysis were recorded over a period of seven years, from 2009 to 2016. This period covers a large fraction of the current (24th) solar cycle, which started in January 2008 

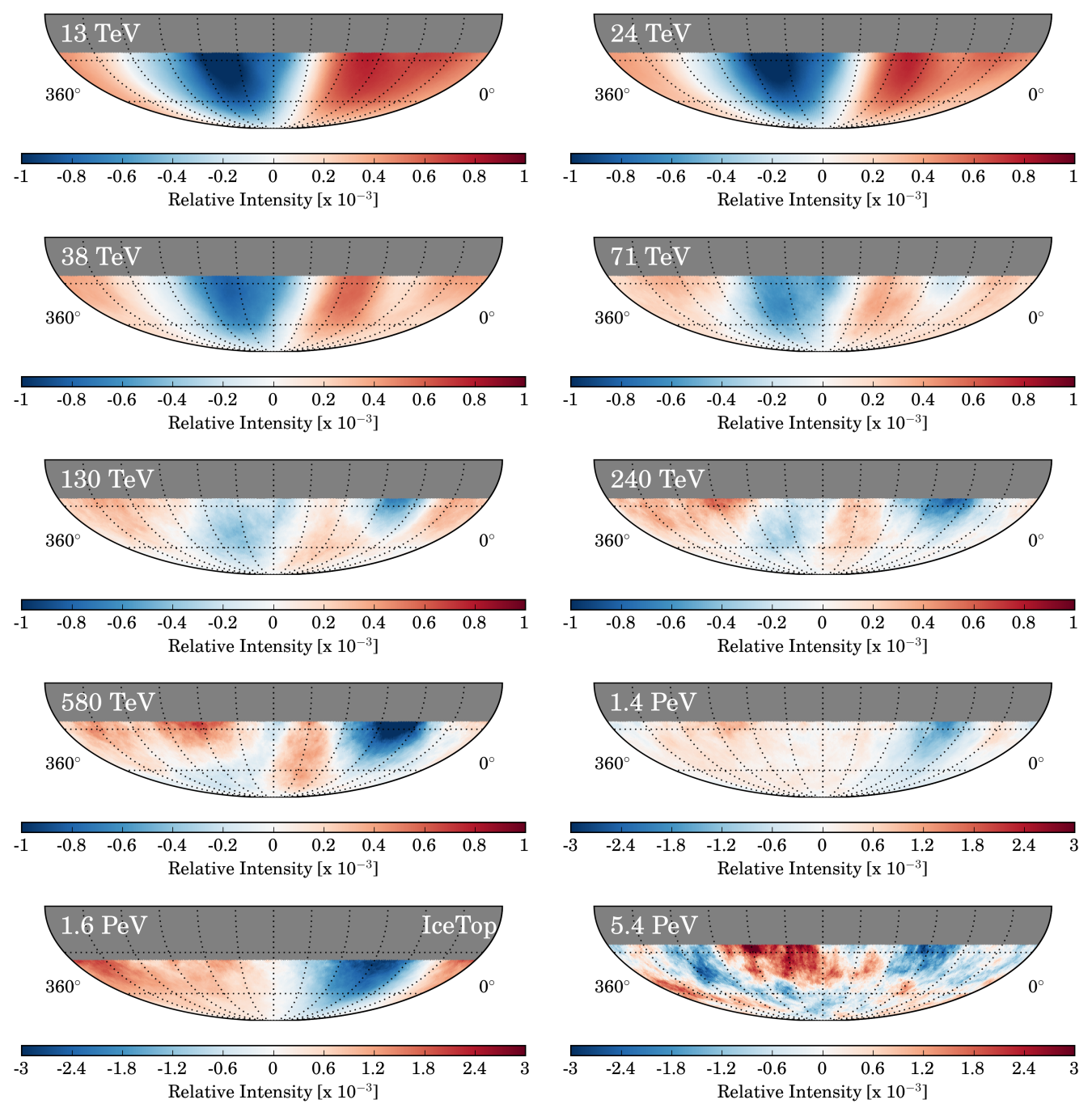

Figure 1: Equatorial maps of the relative intensity of the cosmic-ray flux. An angular smoothing with $20^{\circ}$ radius is applied to all maps. Note that the three highest-energy maps have a different intensity scale.

and reached a maximum in April 2014. Figure 3 shows the one-dimensional projection of the relative intensity in right ascension for each year of data. The yearly data points are placed side by side in time sequence, with the different right ascension bins delineated by vertical lines. The shaded areas indicate systematic errors, estimated using the anti-sidereal frame for each year as described in [13]. We conclude that the large-scale structure is stable over the data period considered here. The Tibet experiment also did not observe significant time variation in the large-scale anisotropy in the northern hemisphere between 1999 and 2008 [20]. In addition, no time-dependence of the largescale anisotropy is seen in data taken with the AMANDA-II detector at the South Pole between 2000 and 2006 [21]. In contrast, Milagro reported an increase in the amplitude of the large-scale structure between 2000 and 2007 [5]. In a separate analysis [13], we also found that the smaller structure shows no significant dependence on time. The ARGO-YBJ experiment also observed a steadiness in the small-scale structure of their measured anisotropy [8]. 

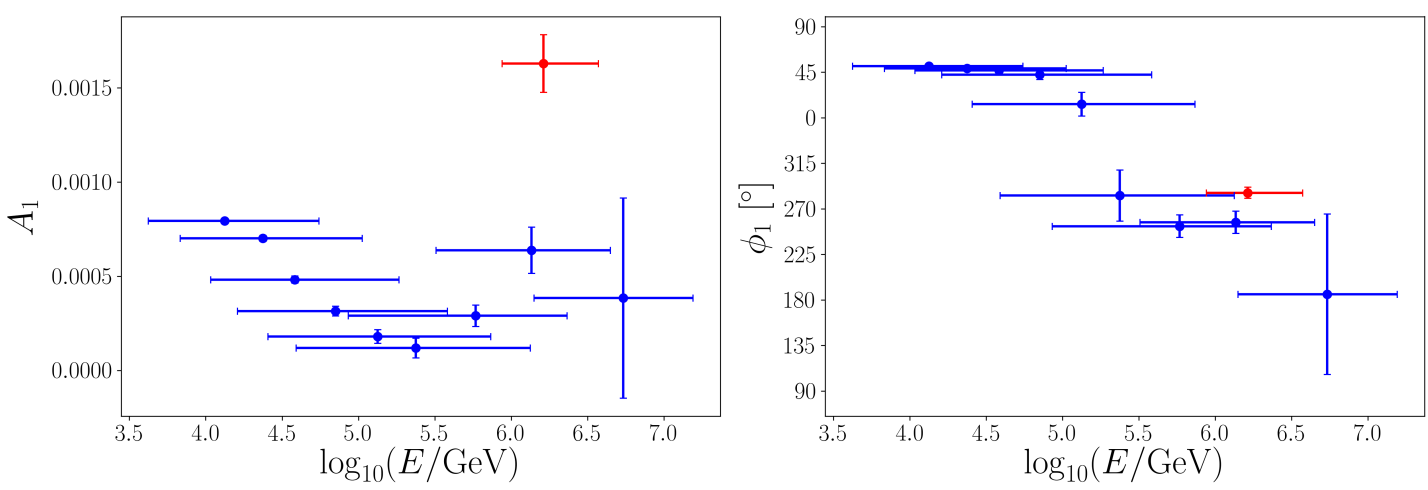

Figure 2: Amplitude (left) and phase (right) of dipole fit to IceCube (blue) and IceTop (red) sky maps for various energy bins. Data points indicate the median energy of each energy bin, with error bars showing the $68 \%$ containment interval.

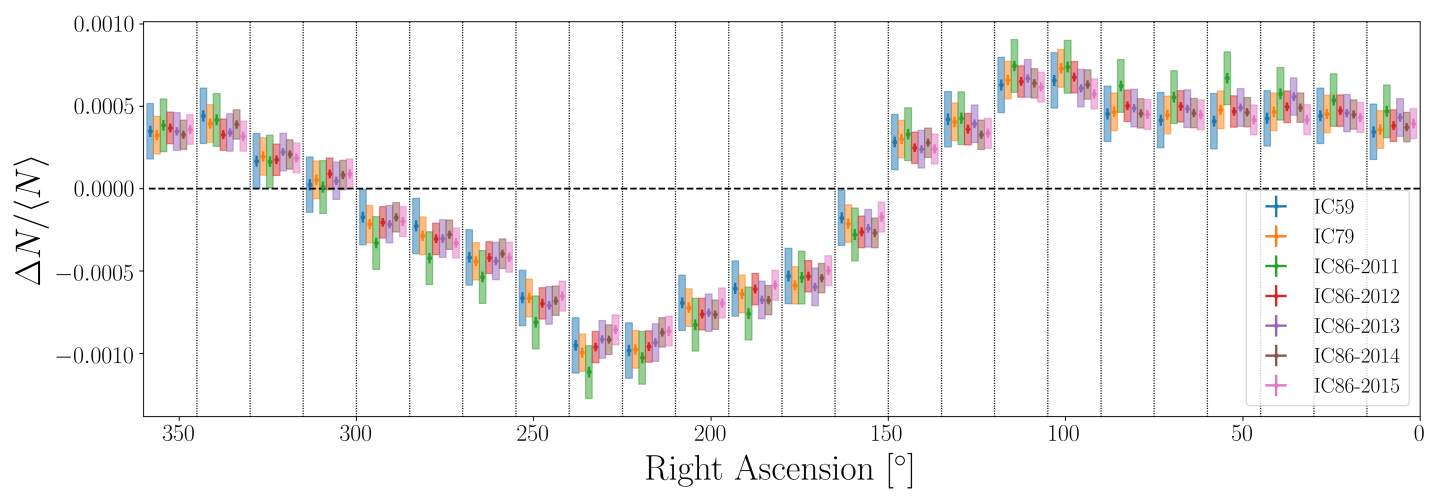

Figure 3: Projection of relative intensity for all declinations as a function of right ascension for each configuration of the IceCube detector from IC59 to the fifth year of IC86. The yearly data points are placed side by side in time sequence, and the different right ascension bins are delineated by vertical lines. The shaded areas indicate systematic errors, calculated using the anti-sidereal frame for each year independently.

\section{Solar Dipole}

An important systematic check of the reliability of the anisotropy analysis is the study of the solar dipole, i.e., the dipole in the cosmic-ray arrival direction distribution caused by the motion of the Earth around the Sun. This dipole appears when the cosmic-ray arrival directions are plotted in a frame where the position of the Sun is at a fixed location. The projection of the relative intensity in right ascension for the sidereal and solar frame are shown in Fig. 4. For the solar frame, the "right ascension" axis shows the difference between the right ascension of the event and the right ascension of the Sun. Note that the Sun is located at $0^{\circ}$ and the direction of motion (and thus the dipole maximum) is at $270^{\circ}$. The fit of the projection to a dipole results in an amplitude of $(2.231 \pm 0.031) \times 10^{-4}$ and a phase of $(267.58 \pm 0.78)^{\circ}$. The $\chi^{2}$-probability of the fit is 0.45 $\left(\chi^{2}=21.60\right.$ for 23 degrees of freedom). The measured amplitude of the projection agrees well with expectations.

Currently, we are studying possible seasonal variations of the solar dipole and the sidereal 


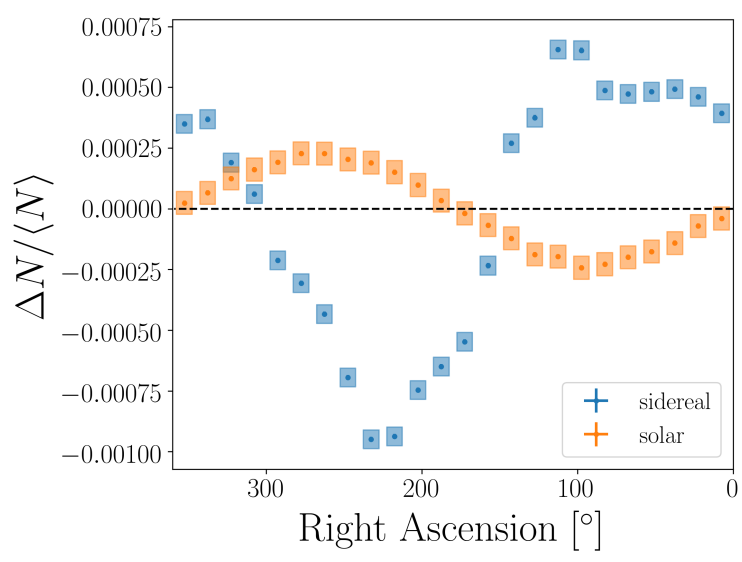

Figure 4: Projection of relative intensity in right ascension for solar and sidereal time. Error boxes indicate systematic errors. For the solar frame, the right ascension axis shows the difference between the right ascension of the event and the right ascension of the Sun.

anisotropy. Seasonal variations in the solar dipole can manifest themselves as an anisotropy in the sidereal frame and vice versa, so this study will help to understand possible systematic effects on the amplitude and phase of the sidereal anisotropy. In addition, physical effects, for example the Compton-Getting effect (apparent dipole due to the relative motion between the solar system and the cosmic-ray rest frame) [22] could manifest themselves in a faint seasonal variation of the solar dipole and the sidereal anisotropy.

\section{Summary and Outlook}

The analysis of seven years of cosmic-ray data observed with the IceCube Neutrino Observatory reveals a strongly energy-dependent anisotropy in the arrival direction distribution. The anisotropy is most significant at the low-order multipoles of the angular power spectrum (dipole, quadrupole, and octupole), but it is observed on scales down to a few degrees, close to the angular resolution of the detector. At the highest energy, around a few PeV, the IceTop air-shower array provides an independent measurement of the anisotropy. The dipole amplitude is significantly larger in IceTop, a discrepancy possibly caused by the different chemical composition of the IceCube and IceTop data sets. Since IceTop provides additional shower parameters that are sensitive to the chemical composition, this effect can be investigated in more detail in the future. We are currently studying whether the anisotropy shows significant differences for data sets containing mostly light (proton and helium) or mostly heavy elements (up to iron).

The IceCube detector only covers parts of the southern hemisphere. In the northern hemisphere, the High Altitude Water Cherenkov (HAWC) Observatory in Mexico now provides cosmicray data at an unprecedented rate [9]. The combination of IceCube and HAWC cosmic-ray data produces a data set that covers almost the entire sky and helps to overcome some of the shortcomings of analyses with partial sky coverage [23]. A combined analysis using HAWC and IceCube data is also presented at this conference [24]. 


\section{References}

[1] Tibet AS $\gamma$ Collaboration, M. Amenomori et al., Large-Scale Sidereal Anisotropy of Galactic Cosmic-Ray Intensity Observed by the Tibet Air Shower Array, Astrophys. J. 626 (2005) L29.

[2] Kamiokande Collaboration, K. Munakata et al., Large-Scale Anisotropy of the Cosmic-Ray Muon Flux in Kamiokande, Phys. Rev. D 56 (1997) 23.

[3] Super-Kamiokande Collaboration, G. Guillian et al., Observation of the Anisotropy of $10 \mathrm{TeV}$ Primary Cosmic Ray Nuclei Flux with the Super-Kamiokande-I Detector, Phys. Rev. D 75 (2007) 062003.

[4] Milagro Collaboration, A.A. Abdo et al., Discovery of Localized Regions of Excess 10-TeV Cosmic Rays, Phys. Rev. Lett. 101 (2008) 221101.

[5] Milagro Collaboration, A.A. Abdo et al., The Large-Scale Cosmic-Ray Anisotropy as Observed with Milagro, Astrophys. J. 69821212009.

[6] EAS-TOP Collaboration, M. Aglietta et al., Evolution of the Cosmic-Ray Anisotropy Above $10^{14} \mathrm{eV}$, Astrophys. J. 692 (2009) L130.

[7] MINOS Collaboration, J.K. deJong et al., Observations of Large Scale Sidereal Anisotropy in 1 and $11 \mathrm{TeV}$ Cosmic Rays from the MINOS Experiment, Proc. 32nd ICRC, Beijing, China (2011) [arXiv:1201.2621].

[8] ARGO-YBJ Collaboration, B. Bartoli et al., Medium Scale Anisotropy in the TeV Cosmic Ray Flux Observed by ARGO-YBJ, Phys. Rev. D 88 (2013) 082001.

[9] HAWC Collaboration, A.U. Abeysekara et al., Observation of Small-Scale Anisotropy in the Arrival Direction Distribution of TeV Cosmic Rays with HAWC, Astrophys. J. 796 (2014) 108.

[10] IceCube Collaboration, R.U. Abbasi et al., Measurement of the Anisotropy of Cosmic-Ray Arrival Directions with IceCube, Astrophys. J. 718 (2010) L194.

[11] IceCube Collaboration, R.U. Abbasi et al., Observation of Anisotropy in the Arrival Directions of Galactic Cosmic Rays at Multiple Angular Scales with IceCube, Astrophys. J. 740 (2011) 16.

[12] IceCube Collaboration, R.U. Abbasi et al., Observation of Anisotropy in the Galactic Cosmic-Ray Arrival Directions at 400 TeV with IceCube, Astrophys. J. 746 (2012) 33.

[13] IceCube Collaboration, M.G. Aartsen et al., Anisotropy in Cosmic-Ray Arrival Directions in the Southern Hemisphere Based on Six Years of Data from the IceCube Detector. Astrophys. J. 826 (2016) 220.

[14] IceCube Collaboration, M.G. Aartsen et al., Observation of Cosmic-Ray Anisotropy with the IceTop Air Shower Array, Astrophys. J. 765 (2013) 55.

[15] Pierre Auger Collaboration, A. Aab et al., Multi-Resolution Anisotropy Studies of Ultrahigh-Energy Cosmic Rays Detected at the Pierre Auger Observatory, submitted to Journal of Cosmology and Astroparticle Physics. [arXiv:1611.06812].

[16] M. Ahlers and P. Mertsch, Origin of Small-Scale Anisotropies in Galactic Cosmic Rays, Progress in Particle and Nuclear Physics, 94 (2017) 184.

[17] IceCube Collaboration, M.G. Aartsen et al., The IceCube Neutrino Observatory: Instrumentation and Online Systems, Journal of Instrumentation 12 (2017) P03012. 
[18] IceCube Collaboration, R.U. Abbasi et al., IceTop: The Surface Component of IceCube, Nucl. Instr. Meth. A 700 (2013) 188.

[19] K.M. Górski et al., HEALPix - a Framework for High Resolution Discretization, and Fast Analysis of Data Distributed on the Sphere, Astrophys. J. 622 (2005) 759.

[20] Tibet AS $\gamma$ Collaboration, M. Amenomori et al., On Temporal Variations of the Multi-TeV Cosmic Ray Anisotropy Using the Tibet III Air Shower Array, Astrophys. J. 711 (2010) 119.

[21] IceCube Collaboration, M.G. Aartsen et al., Study of the Time-Dependence of the Cosmic-Ray Anisotropy with AMANDA and IceCube, Proc. 33rd ICRC, Rio de Janeiro, Brazil (2013) [arXiv:1309.7006].

[22] A.H. Compton and I.A. Getting, An Apparent Effect of Galactic Rotation on the Intensity of Cosmic Rays, Phys. Rev. 47 (1935) 817.

[23] M. Ahlers, S.Y. BenZvi, P. Desiati, J.C. Díaz-Vélez, D.W. Fiorino, and S. Westerhoff, A New Maximum-Likelihood Technique for Reconstructing Cosmic-Ray Anisotropy at All Angular Scales, Astrophys. J. 823 (2016) 10.

[24] IceCube Collaboration, J.C. Díaz-Vélez, PoS ( ICRC2017) 539 (these proceedings). 